S. Horsch, G. Torsello (Hrsg.) Gefäßchirurgie kontrovers 
S. Horsch - G. Torsello

Herausgeber

\section{Gefäßchirurgie kontrovers}

Empfehlenswertes

und Nichtbewährtes 
Prof. Dr. med. Svante Horsch

Klinik für Allgemein- und Gefäßchirurgie

Krankenhaus Porz am Rhein

Urbacher Weg 19

51149 Köln

Prof. Dr. med. Giovanni Torsello

Abt. für Gefäßchirurgie

St. Franziskus Hospital

Hohenzollernring 72

48145 Münster

ISBN 978-3-7985-1238-2

ISBN 978-3-642-53826-1 (eBook)

DOI 10.1007/978-3-642-53826-1

Die Deutsche Bibliothek - CIP-Einheitsaufnahme

Ein Titeldatensatz für diese Publikation

ist bei der Deutschen Bibliothek erhältlich

Dieses Werk ist urheberrechtlich geschützt. Die dadurch begründeten Rechte, Insbesondere die der Übersetzung, des Nachdrucks, des Vortrags, der Entnahme von Abbildungen und Tabellen, der Funksendung, der Mikroverfilmung oder der Vervielfältigung auf anderen Wegen und der Speicherung in Datenverarbeitungsanlagen, bleiben, auch bei nur auszugsweiser Verwertung, vorbehalten. Eine Vervielfältigung dieses Werkes oder von Teilen dieses Werkes ist auch im Einzelfall nur in den Grenzen der gesetzlichen Bestimmungen des Urheberrechtsgesetzes der Bundesrepublik Deutschland vom 9. September 1965 in der jeweils geltenden Fassung zulässig. Sie ist grundsätzlich vergütungspflichtig. Zuwiderhandlungen unterliegen den Strafbestimmungen des Urheberrechtsgesetzes.

(c) Springer-Verlag Berlin Heidelberg 2000

Originally published by Steinkopff-Verlag Darmstadt in 2000

Die Wiedergabe von Gebrauchsnamen, Handelsnamen, Warenbezeichnungen usw. in diesem Werk berechtigt auch ohne besondere Kennzeichnung nicht zu der Annahme, daß solche Namen im Sinne der Warenzeichen- und Markenschutz-Gesetzgebung als frei zu betrachten wären und daher von jedermann benutzt werden dürften.

Produkthaftung: Für Angaben über Dosierungsanweisungen und Applikationsformen kann vom Verlag keine Gewähr übernommen werden. Derartige Angaben müssen vom jeweiligen Anwender im Einzelfall anhand anderer Literaturstellen auf ihre Richtigkeit überprüft werden.

Verlagsredaktion: Dr. Annette Gasser - Herstellung: Heinz J. Schäfer

Umschlaggestaltung: Erich Kirchner, Heidelberg

Satz: Typoservice, Griesheim

Gedruckt auf säurefreiem Papier 


\section{Vorwort}

Das 13. Titisee-Symposium setzt zweifelsohne die Tradition der vorangegangenen zwölf Symposien fort. Dies bedeutet zum einen hochaktuelle, interessante Kongreßthematik und zum anderen herausragende Referenten.

Der Titel des 13. Titisee-Symposiums lautet „Gefäßchirurgie kontrovers - Empfehlenswertes und Nichtbewährtes“. Zu keinem Zeitpunkt wurde die Gefäßchirurgie so kontrovers diskutiert wie heute. Noch nie war es so schwer, einen Konsens über Empfehlenswertes und Nichtbewährtes zu finden.

Die Kongreßthematik umfaßt neben dem Eröffnungsreferat von Prof. Dr. Müller-Wiefel den klinischen Aspekt dieser Kontroversität sowie administrative Fragen bzw. Fragen des Managements in der Gefäßchirurgie. Hierbei wird die schwierige Situation eines Arztes in der Position des Leiters einer Abteilung für Gefäßchirurgie analysiert. Außerdem wird über die Organisation und Optimierung der Abläufe in der Klinik, die Harmonisierung des Verhältnisses mit der Krankenhaus-Administrative, den Umgang mit den Kostenträgern und die richtigen Budgetierung gesprochen. Es folgen Referate über Mitarbeiterorganisation, Motivationsstrategie, Geräteinvestitionen und schließlich über die Eigenschaften eines leitenden Arztes.

Der wissenschaftliche Teil behandelt Themen der zukünftigen, aber nicht weniger kontrovers diskutierten Innovationen in der Gefäßchirurgie wie endovaskuläre Aneurysmabehandlung, laparoskopische Aortenchirurgie und endovaskuläre Therapie der Carotisstenose. Das Symposium wird mit dem Forum aktuell abgerundet. Darin kommen Fragen zu medizinisch-rechtlichen und medizinisch-ethischen Aspekten zur Sprache.

Das Titisee-Symposium hat sich zu einer institutionellen, wissenschaftlichen und gleichzeitig praxisnahen Veranstaltung entwickelt. Wir hoffen, daß die gewählte Thematik des 13. Symposiums zu interessanten, kontroversen und lehrreichen Diskussionen beiträgt und uns durch die Praxisnähe der Thematik hier und dort in unserem klinischen Alltag helfen wird.

Der Firma Boston Scientific ist zu danken, daß sie dieses sowohl für den Arzt als auch für seinen Patienten sehr wertvolle Symposium ermöglicht. 


\section{Inhaltsverzeichnis}

\section{Carotischirurgie}

Gefäßchirurgie: Gestern, Heute und in Zukunft

Müller-Wiefel, H.

Sind die präoperative Angiographie und Computertomographie verzichtbar?

Schwilden, E.-D. . . . . . . . . . . . . . . . . . . . . . . . . . . . .

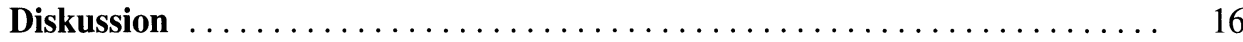

Eversion oder klassische TEA - Welche Operationstechnik ist zu bevorzugen?

Schunn, C., D. Raithel ............................... 19

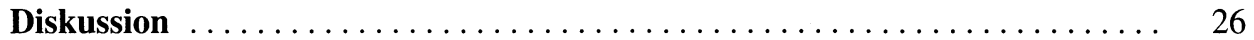

Soll die OP beim neurologisch instabilen Carotispatienten Routine werden?

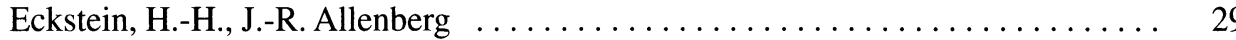

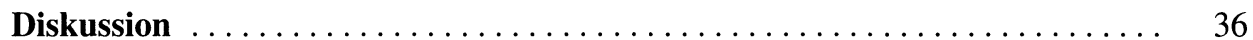

\section{Chronisch arterielle Verschlußkrankheit}

Die periphere arterielle Verschlußkrankheit (pAVK) aus angiologischer Sicht Scheffler, P., J. Groß . . . . . . . . . . . . . . . . . . . . . . . . . . . . . . . . . . .

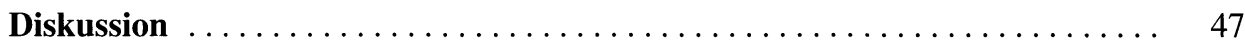

Aktuelle und zukunftsweisende Aspekte der interventionellen Therapie der pAVK

Roth, F.J., S. P. Roth . . . . . . . . . . . . . . . . . . . . . . . . 49

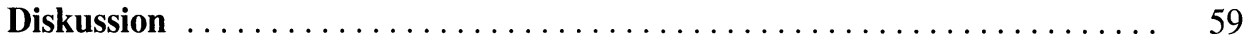

Aorto-iliakale Revaskularisationen: Interventionell, operativ oder kombiniert? Steckmeier, B., F. Verrel, U. Szeimies

Femoropopliteale Rekonstruktion: Interventionell, operativ oder kombiniert? Ktenidis, K., S. Horsch . . . . . . . . . . . . . . . . . . . . . . . . . . . . . 71 
Kruro-pedale Rekonstruktionen bei der chronisch arteriellen

Verschlußkrankheit: Operativ, interventionell oder kombiniert?

Klenk, E., G. Torsello

Diskussion

\section{Varikosis und Diabetischer Fuß}

Moderne gefäßchirurgische Vorgehensweise beim (neuro)ischämischen diabetischen Fuß-Syndrom

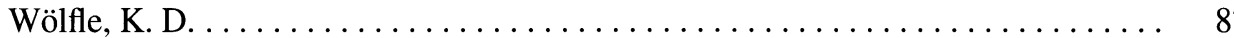

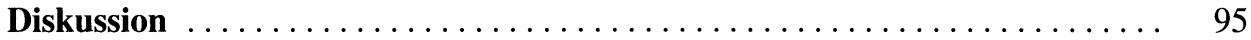

\section{Position der Leiter der Abteilung für Gefäßchirurgie}

Harmonisierung der Verhältnisse zwischen Fachabteilung und Krankenhausadministrative

Niedermeier, H.-P.

Umgang mit den Kostenträgern

Jost, J. O

Diskussion

Mitarbeiterorganisation - Motivationsstrategien, Delegationsmöglichkeiten

Betzler, M.

Diskussion

Öffentlichkeitsarbeit

Hupp, T., A. M. Endisch

Diskussion

Geräteinvestition: Top oder Flop?

Balzer, K.

Diskussion

Was muß ein leitender Arzt können?

Fraedrich, G. 


\section{Zukunftsträchtige Innovationen in der Gefäßchirurgie}

Endovaskuläre Therapie des infrarenalen Aortenaneurysmas

Umscheid, T., W. J. Stelter, P. Ziegler . . . . . . . . . . . . . . . . . . . 149

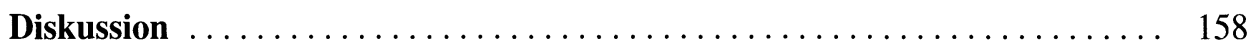

Laparoskopische Gefäßchirurgie

Mumme, A., M. Kemen, L. Barbera $\ldots \ldots \ldots \ldots \ldots \ldots \ldots \ldots \ldots \ldots \ldots$

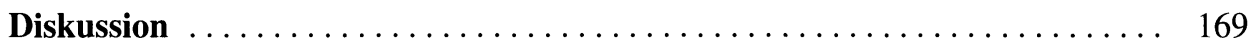

Die endovaskuläre Therapie der Carotisstenose

Gißler, H. M., K. Mathias . . . . . . . . . . . . . . . . . . . . . 171

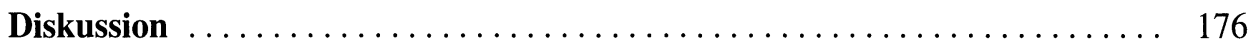

\section{Forum aktuell}

Medizinisch rechtliche Aspekte:

Arzthaftung, Behandlungsabbruch am sterbenden Patienten

Zurstraßen, A.

Medizinisch-ethische Aspekte an der Schwelle des 21. Jahrhunderts

Abel, M.

Diskussion 